\title{
Problem formulation in the environmental risk assessment for genetically modified plants
}

\author{
Jeffrey D. Wolt · Paul Keese - Alan Raybould • \\ Julie W. Fitzpatrick · Moisés Burachik · Alan Gray • \\ Stephen S. Olin · Joachim Schiemann · Mark Sears • \\ Felicia Wu
}

Received: 3 March 2009/Accepted: 28 August 2009/Published online: 15 September 2009

(C) The Author(s) 2009. This article is published with open access at Springerlink.com

\begin{abstract}
Problem formulation is the first step in environmental risk assessment (ERA) where policy goals, scope, assessment endpoints, and methodology are distilled to an explicitly stated problem and approach for analysis. The consistency and utility of ERAs for genetically modified (GM) plants can be
\end{abstract}

A Report from the ILSI Research Foundation Expert Consultation on Problem Formulation for ERA of GM Plants.

\section{J. D. Wolt}

Biosafety Institute for Genetically Modified Agricultural

Products, Iowa State University, Ames, IA, USA

e-mail: jdwolt@iastate.edu

\section{P. Keese}

Department of Health and Ageing, Biotechnology

Regulatory Services, Office of Gene Technology

Regulator, Canberra, ACT, Australia

e-mail: Paul.Keese@health.gov.au

\author{
A. Raybould \\ Syngenta, Berkshire, UK \\ e-mail: alan.raybould@syngenta.com
}

J. W. Fitzpatrick $(\bowtie) \cdot$ S. S. Olin

ILSI Research Foundation, 1156 15th Street,

NW, Washington, DC 20005, USA

e-mail: juliefitzpatrick@verizon.net

\section{S. S. Olin \\ e-mail: solin@ilsi.org}

M. Burachik

Oficina de Biotecnología, Secretaría de Agricultura,

Buenos Aires, Argentina

e-mail: mburac@minprod.gov.ar improved through rigorous problem formulation (PF), producing an analysis plan that describes relevant exposure scenarios and the potential consequences of these scenarios. A properly executed PF assures the relevance of ERA outcomes for decision-making. Adopting a harmonized approach to problem

\author{
A. Gray \\ Centre for Ecology \& Hydrology, Dorset, UK \\ e-mail: ajg@ceh.ac.uk
J. Schiemann
Julius Kuehn Institute, Federal Research Centre for
Cultivated Plants, Quedlinburg, Germany
e-mail: joachim.schiemann@jki.bund.de
M. Sears
Department of Environmental Biology,
University of Guelph, Ontario, Canada
e-mail: msears@uoguelph.ca

\section{F. Wu}
Department of Environmental \& Occupational Health,
University of Pittsburgh, Pittsburgh, PA, USA
e-mail: few8@pitt.edu


formulation should bring about greater uniformity in the ERA process for GM plants among regulatory regimes globally. This paper is the product of an international expert group convened by the International Life Sciences Institute (ILSI) Research Foundation.

Keywords Ecological risk assessment . GMO · Genetically engineered · Hazard identification

\section{Introduction}

Risk assessment is widely used in decision-making concerning the release of genetically modified (GM) plants into the environment (EFSA 2006). The process of integrating the likelihood and consequences of exposure, in terms of harm, forms the basis of environmental risk assessment (ERA). As the first step in ERA, the problem formulation (PF) establishes the parameters that are of greatest relevance to the assessment.

A variety of national, regional, and international approaches to ERA of GM plants are emerging (Hill 2005), and these contain differing legislative triggers, terminology, and guidance regarding how the assessments are to be performed. The apparent differences among various assessment protocols obscure their similar underlying principles of case-by-case comparative risk assessment. Clarifying these underlying principles can lead to clearer assessments and improved communication among interested and affected parties. Recognizing common principles for PF will encourage harmonized approaches for risk assessment and may help less developed countries to formulate effective and relevant biosafety regulations for GM plants.

This paper proposes a common PF framework for environmental risk assessment of GM plants (Fig. 1). The framework does the following: (i) it provides a common language for the evaluation and communication of similarities and differences among various assessment regimens (see box-Glossary of Terms); (ii) it affords the necessary flexibility for further evolution and improvement of assessments and their harmonization; (iii) it offers a template for environmental assessment that may be applied in emerging national or regional regulatory guidance; and (iv) it aligns with the principles outlined in international conventions such as the Cartagena Protocol on
Biosafety (http://www.cbd.int/biosafety/protocol.shtml) and the phytosanitary standards of the International Plant Protection Convention (IPPC 2001). The ERA paradigm described by the US Environmental Protection Agency (EPA) (USEPA 1992, 1998) has been used by the authors as a conceptual and procedural basis for a common framework and terminology that can be applied to ERAs for GM plants.

An inadequate PF may compromise the entire ERA and add to the level of uncertainty in subsequent decision-making. Frequent outcomes of this type of failure are continuing requests for more data, disproportionate risk mitigation measures and miscommunication of risk findings; this results in increased concerns about the environmental impact (Johnson et al. 2007; Raybould 2006) and leads to delayed decision-making. Some authors contend that such delays may lead to increased negative environmental impacts because of the consequent delays in the introduction of environmentally beneficial products (Raybould 2006, 2007). Additionally, an ERA with a poorly developed PF may have inadequately specified or inappropriate expressions of the environmental value to be protected (benefits including processes by which the environment produces resources), or insufficient clarity regarding the purpose and use of the data being collected. This report presents a framework for constructing PFs that can be applied to ERAs for GM plants.

\section{Problem formulation framework}

The first step in ERA is problem formulation (USEPA 1998), which has also been referred to as hazard identification (Hill 2005; OECD 2003). We use the term problem formulation because it better reflects the broad base of information regarding the type and nature of potential adverse effects considered in an ERA for GM plants. A generic framework for $\mathrm{PF}$ is described in this section and shown in Fig. 1, recognizing that the case-by-case and comparative nature of ERAs for GM plants requires that many aspects of the problem be shaped by casespecific considerations as detailed in Developing the Problem Formulation. At the core of the PF process is the establishment of the ERA's parameters (problem context) and the identification of risks of greatest relevance (problem definition). 
Fig. 1 Problem formulation within the paradigm for environmental risk assessment (ERA). The problem context develops the parameters and identifies constraints for the ERA, which may arise from legal statutes and institutional guidelines. Problem definition shapes the ERA into a manageable form for analysis through consideration of the casespecific attributes of the GM crop being assessed, identification of logically relevant concerns, and description of cause-effect relationships

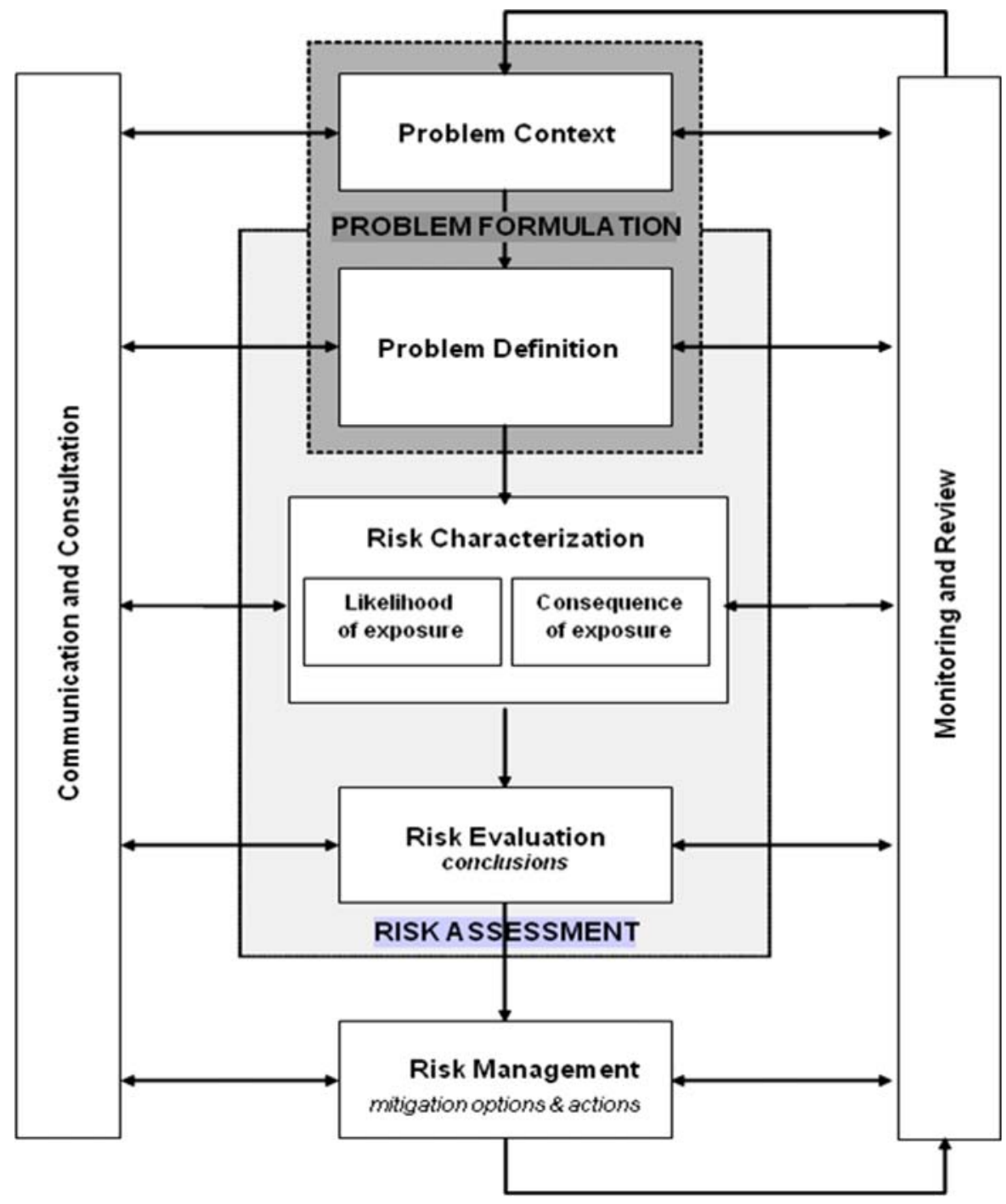

Problem context

The problem context for risk assessment reflects values derived from the broad environmental policies and goals that direct risk analysis. Establishing the problem context sets the parameters for the risk assessment, including; protection goals, environmental scope, standard assessment endpoints (Suter 2000), and assessment methodology (see Glossary of Terms for definitions). In addition, the problem context describes case-specific details of the GM crop and certain baseline information used to determine the relative risk that can be attributed to the modification. This baseline information can include details of the biology of the parent organism and the nature of the receiving environment (e.g., presence of sexually compatible relatives, agronomic practices, presence of nearby protected areas or species, climate, etc.).

Environmental risk assessments are initiated to address protection goals, which may be defined in law, statutes, regulations, or guidance. Therefore, the problem context may include problems and endpoints for analysis with varying levels of relative importance or relevance to the specific case that is ultimately addressed in a particular ERA. In some cases, the problem context is determined by the purpose and scope of the ERA as described in regulatory standards. Such standards may also prescribe the characteristics of an appropriate comparator, general methodology to be used, and criteria for distinguishing between 


\section{Glossary of terms}

Assessment endpoint-An explicit expression of the environmental value that is to be protected. Operationally, it is defined by an identified environmental entity of value that is susceptible to harm and an attribute that provides evidence of harm. For example, beneficial insects are valued ecological entities; abundance within the agroecosystem is an important attribute; "beneficial insect abundance" constitutes an assessment endpoint.

Environmental risk assessment (ERA)—The process of identifying significant risks to the environment, estimating the level of risk, and determining those risks that require measures to reduce the level of risk (USEPA 1998).

Environmental value - The beneficial uses of the environment including the processes by which the environment produces resources.

Exposure-The contact or co-occurrence of a changed attribute of a GM plant with an environmental entity of value.

Exposure scenario-A particular set of circumstances describing the opportunity for harm to an environmental entity of value.

Harm-A negative outcome or effect of an action or event (=adverse effect).

Measurement endpoint-A measurable response to the changed attribute of the plant that is quantifiably related to the assessment endpoint (USEPA 1998).

Phenotype - Observable characteristics of an organism described as physical or biochemical traits of an organism. For the purpose of ERA, what constitutes a phenotype should reflect biologically relevant level of detail consistent with the particular risk comparison being made.

Postulated risk-A potential harm that may manifest from a plausible exposure scenario and is subject to further analysis.

Problem context-The activity that establishes the parameters for the risk assessment, including policy goals, scope, assessment endpoints, and methodology.

Problem definition-The activity that leads to the identification of postulated significant risks that warrant further analysis for a specific ERA case and which leads to a specified analysis plan.

Problem formulation - The first step in ERA whereby policy goals, scope, assessment endpoints, and methodology are developed into an explicitly stated problem and an approach for analysis; comprised of the problem context and problem description.

Risk hypothesis-A tentative explanation taken to be true for the purpose of argument or investigation. This should not be confused with scientific hypotheses, which are specific, testable postulates that will be part of the analytical phase of the ERA.

Scope-The state of the environment in which a situation exists.

Uncertainty-A form or source of doubt.

meaningful and negligible differences (Australia 2000; Standards Australia 2004). For example, the term 'environment' may require operational definition as the scope of the ERA may need to address anthropogenic as well as natural components of environment in some jurisdictions (New Zealand 1996) but not in others (Australia 2000). Existing guidance documents addressing current risk assessment and risk management practices should be considered in the problem formulation process.

Importantly, the risk assessor must refine a broadly stated issue or concern into a relevant and manageable analysis. For instance, the stated protection goal may be to provide an adequate level of protection of biological diversity, http://www.cbd.int/biosafety/ protocol.shtml. A critical challenge of PF is to identify an observable, measurable property that adequately reflects this desirable quality. To achieve this, it is necessary to define assessment endpoints and methodology that will direct the characterization of risk and produce information that will be relevant for decision-making. For example, beneficial insects are valued ecological entities and their abundance within the agroecosystem is important and can serve as a proxy or indicator of biological diversity. Therefore, "beneficial insect abundance" constitutes a useful assessment endpoint.

\section{Problem definition}

Problem definition is a distilling exercise that leads to the identification of reasonably postulated risks that warrant further analysis, and removes from consideration other potential but negligible risks. This is done in the form of a scoping assessment that generates and evaluates potential exposure scenarios (Garcia-Alonso et al. 2006). Each exposure scenario represents a meaningful problem that describes a causally linked pathway, or set of circumstances, that lead from the environmental release of the GM plant through to an environmental entity of value that may be adversely affected. Exposure when causally linked 
to harm allows for the description of risk as an adverse consequence from exposure.

In distilling the problem to a relevant form for analysis, the problem definition considers the protection goal and the specific case; this encompasses the plant being modified (the appropriate comparator), the nature of the GM plant, and the environment in which exposure is likely. Initially, this is a mental exercise or an abbreviated assessment. Experience with both GM and non-GM plants helps to identify the potentially meaningful problems. No single PF is likely to address every concern; rather, each concern that is eventually deemed relevant to the GM plant will be subject to a specific PF within the ERA.

The degree to which concerns are addressed within a given assessment (such as a regulatory dossier) will likely vary from case to case. Many concerns or questions of risk may be readily answered on the basis of prior knowledge and this prior knowledge may rule out some scenarios as insubstantial. For instance, for maize planted in the European Union, potential harm resulting from pollen-mediated gene flow to a sexually compatible wild relative is not a problem that requires analysis, due to the absence of wild relatives. The degree to which the ERA formally poses hypotheses and tests them with prior data varies depending on how and by whom the risk assessment will be used.

Many of the concerns included in the problem definition for a GM plant are general to all ERAs, and descriptions may be found in legislation, policy papers, guidance documents, and descriptions of existing risk analysis/management frameworks. In addition to general concerns, features that are specific to each particular case should be documented in each $\mathrm{PF}$. These include consideration of the specific GM plant event, the environment where it is released, and practices associated with its use. It is therefore necessary to evaluate whether harm could arise from a changed plant attribute by asking several questions: What must happen for harm to occur? Is there a reasonable causal pathway to harm? What is the seriousness and likelihood of harm? Hypotheses of no harm form the basis of a hypothesis-driven approach to addressing these questions.

Thus, problem definition will achieve the following: (1) select relevant assessment endpoints based on policy objectives, (2) postulate reasonable exposure scenarios that might result in environmental harm arising from an activity or use of the GM plant or its processed form, and (3) identify those scenarios that merit detailed risk characterization. Addressing problem definition in the manner detailed in this paper seeks to reduce the development of policies which do not identify and address risks appropriately and may become de facto barriers to the use of GM plants.

The definition of harm associated with GM plant release should relate to commercial and regulatory intent. For instance, the UK Farm Scale Evaluation Trials (http://www.defra.gov.uk/environment/gm/fse/ ) considered how GM cropping systems compared to current cropping practice in the United Kingdom and assessed whether the use of broad-spectrum herbicides with GM plants would reduce the levels of farmland biodiversity (including weeds). The improved weed control from the pattern of use of the herbicide in two of the three GM cropping systems examined was described as 'harmful'. Such a characterization of harm is meaningful only to the extent that it is consistent with the regulatory intent under which the assessment is conducted.

\section{Exposure}

Exposure is the contact or co-occurrence of the modified attribute of the GM crop with an environmental entity of value. For instance, for a GM plant expressing an insect resistance trait and co-occurring in a given environment with a presumed sensitive organism (the entity of value), there must be a plausible pathway for exposure of the organism to the modified plant attribute (an insect toxin in this example). The description of this pathway represents an exposure scenario. Thus, for Lepidoptera-active Cry 1 proteins expressed in maize pollen, the ingestion of pollen occurring on milkweed in and around maize fields by a monarch butterfly is considered a reasonable exposure scenario for ERA (Sears et al. 2001; Wolt et al. 2003).

Management of the GM plant-including activities such as growing, propagating, breeding, producing, processing, importing, transporting, disposing, and using-will influence development of exposure scenarios. Worst-case assumptions regarding exposure and the consequences of exposure are frequently used in early stages of ERA to focus on substantive risks that warrant further consideration. When worstcase considerations indicate the possibility of harm, 
there is a need to reformulate the problem to determine if the concern remains under more realistic conditions. Conversely, a finding of minimal harm in a worst-case scenario would allow for a reasonably and conservatively stated finding of no likely harm under realistic conditions of environmental release. For example, a worst-case risk assessment for introgression of virus resistance from transgenic Brassica napus into wild brassica populations shows $B$. nigra and B. napus will hybridize when pollinated manually (Raybould and Cooper 2005), which requires reformulation of the problem to consider whether hybridization will occur spontaneously. The opportunity for spontaneous hybridization in this case is very low, so there is no likely harm under field conditions. For any given case, there thus needs to be a consideration of whether the potential harm arising from exposure is sufficiently uncertain as to require further analysis of exposure and its consequence.

Lack of a reasonable exposure pathway can result in a determination of negligible risk, since without exposure there is no opportunity for harm. A variety of means may be used to postulate reasonable scenarios. Standard problem definition methods (Hayes et al. 2007) may be as simple as using checklists or brainstorming, but they may also extend to more rigorous and systematic methods (Hayes et al. 2004). The nature and formality of the exercise will be case dependent and will reflect preferences and approaches of the responsible authority. Regardless of the approach used, transparency is important to all of the interested and affected parties.

\section{Reasonable risks}

The standard for any ERA should be focusing on reasonably postulated risk to support effective and efficient decision making. Of the many hypothetical risks that can be described during problem definition, most can be readily characterized as negligible. For a few the probable magnitude or significance of harm may require more detailed consideration which involves further elaboration of the exposure scenario into a general statement of concern and a sequence of events linking exposure to a consequence arising from exposure, as shown in the Bacillus thuringiensis (Bt) maize and monarch butterfly risk assessment (Sears et al. 2001).

\section{Developing the problem formulation}

In developing the $\mathrm{PF}$, the overarching protection goals presented in law, regulation, or guidance must be focused into case-specific objectives with clearly defined assessment endpoints and risk hypotheses. These lead within the problem definition to development of the conceptual model and translation into an analysis plan focused on specific testable hypotheses and relevant measurement endpoints.

\section{Assessment endpoints}

The starting point for the problem definition is selection of assessment endpoints relevant to the specific case under consideration, which is an outgrowth of the problem context, as outlined above. The assessment endpoint is an explicit expression of environmental value and is operationally defined as an environmental entity of value susceptible to harm, such as a certain species, and an attribute that provides evidence of harm, such as population size (USEPA 1998). Examples of assessment endpoints typically used in ERA for GM plants are abundance of beneficial organisms in a crop field and population size of wild relatives of the GM plant; while neither is direct evidence of harm per se, substantive changes in these attributes would be cause for further detailed assessment. The ecological entities of value and their measurable attributes should also be recognized in a conceptual model.

\section{Risk hypotheses}

Risk hypotheses arise from the consideration of potentially significant risks. The risk hypothesis is used for clarification and articulation of the relationships identified through problem definition (USEPA 1998). A risk hypothesis stands as an assumption regarding the cause-effect relationships among changed attributes, sources, exposure routes, endpoints, responses, and measures relevant to the ERA. In the analysis phase of the ERA, the risk hypothesis is translated into one or more experimental hypotheses that can be used for testing and corroboration. The use of risk hypotheses can increase clarity in defining and testing postulated risks as well as transparency in communicating the intended purpose of a given ERA. 
The nature of the plant being modified

One of the pitfalls of ERA that can be prevented by $\mathrm{PF}$ is treatment of the plant as a theoretical entity. The PF defines the plant being modified in tangible terms based on the plant's biology.

\section{Defining and identifying differences that may plausibly lead to harm}

Among the factors that should be considered in problem definition, the nature, magnitude, and significance of the changes in the GM plant are of primary importance since they will direct the course of actions required for risk characterization. For instance, modification involving introduction of a single trait like a Cry 1 protein allows for a focused consideration of cause (exposure to Cry1 through pollen release in a given environment) and effect (harm or toxic effects occurring to an entity of value, such as a butterfly). In the case of multiple or stacked traits, PF considers each trait, e.g. Cry1 and Cry3, independently as well as the potential for the traits to interact. Instances in which unintended changes are detected by comparison to a baseline and sufficiently differ from the baseline may require further analysis. Or an early-stage decision may be made to discontinue development of the GM plant if the unintended change represents an undesirable phenotype.

To date, the vast majority of phenotypes that have been assessed have been traits for herbicide tolerance and insect resistance. As other phenotypes are developed, the PF may need to consider more complex changes and the possibility of unintended or unexpected consequences to the modification. Transcription factors and mechanisms that affect metabolic profiles, e.g., RNAi, are examples of newer traits that involve less defined modes of action. In these cases, a sound model for the PF is essential to link an intended change to a postulated harm through plausible pathways of exposure and its adverse effects. Phenotypic alterations that may arise from these molecular level changes, and which may plausibly lead to harm, will need to be identified in the PF process (Nickson 2008).

Comparative risk assessment is a fundamental principle of GM plant ERA. As described in the problem context phase, the endpoint measurement for
GM plants is made relative to comparator plants and cropping practices. Biologically meaningful differences observed between the GM plant and its comparators are attributed to the outcome of the genetic modification. The risk will be evaluated comparatively; i.e., on the basis of a hypothesis of no biologically meaningful differences that could produce an adverse effect on the assessment endpoint associated with the GM phenotype versus the nontransformed comparator, or on the basis of no environmentally relevant difference in the system where the GM phenotype is released. Therefore, it is necessary to establish the basis for comparability and parameters to identify meaningful changes in the transformed plant. The PF seeks relevant existing information describing the environmentally meaningful case-specific attributes of the GM plant under conditions of its release, which will determine the nature and scope of the ERA. The types of information that should be considered at this stage are existing peer reviewed literature, information generated during product development, and previous regulatory decisions and assessments. In the case of GM plants without an unmodified counterpart, a direct comparator does not exist but indirect comparators will exist in the process or ecosystem where the new plant will occur.

All available knowledge should be applied in order to identify meaningful differences for analysis. Knowledge of the trait, the host, and the host-trait combination may be found in existing information. Further information may also be available that describes the gene stability, trait selectivity, level of expression, and mode of action of the change that is manifested. This precursor information will typically establish the comparability of the transformed event relative to a near isoline as well as to the normal range of a component seen in the crop. Information of this type is commonly required in the overall preparation of regulatory dossiers for GM plants and is frequently available in advance of the ERA; it thus allows for the ERA to be shaped in a way that is relevant to the case being studied. This information alone does not establish harm or lack thereof, but it does establish the properties of the intended change. Importantly, the precursor information should help define those environmental entities most likely to be sensitive to the change manifested in the GM plant. 


\section{Likely environmental interactions}

The type of environmental release determines the intended use, scale, and likely location of GM plants within a receiving environment. Three types of releases applicable to GM plants are confined field trials, incidental release in the course of transit (typically an outcome of moving commodities to processing facilities), and unconfined commercial release. The specific environmental concerns considered and the data needed may differ for a small-scale field trial that is subject to confinement measures versus a wide-scale unconfined release because mitigating factors are used (e.g., buffer zones and field offset distances in the case of confined field trials). The ways in which environmental concerns are addressed for a cultivated crop may be different than for an imported grain (incidental release).

Location in space and time, especially as it relates to environmental entities of value, will have a strong bearing on the development of exposure scenarios. In some cases, it may be useful to distinguish different land use types (e.g., urban, natural vegetation, agriculture, and aquatic) that will be relevant to the ERA. Although the GM plant may occur in several land use types, the focus of concern is typically the agroecosystem and its immediate surroundings and environments subject to special protections (such as habitats for threatened or endangered species). Therefore, when identifying specific attributes of assessment endpoints, it is important to draw clear distinctions among the environmental impacts in farmed, unfarmed, or semi-natural environments. This has not been done in much of the discussion of 'escapes' and 'invasions' to date. For instance, almost all examples of invasive hybrids (Ellstrand et al. 1999) are arable weeds and consequently pose a potential problem for agronomy, but they will not necessarily be of direct concern for native species or plant community conservation. For example, B. napus can establish more readily in disturbed habitats and is therefore more likely to have an adverse effect in agricultural environments than in native plant communities.

\section{Defining exposure in the PF}

Potential biotic and abiotic interactions may be important to the PF. Most relevant in this regard is the description of the environmental fate of unique products expressed in the GM plant. Together with environmental fate, expression will determine the potential for environmental persistence and accumulation, and thus exposure. For example, a decline in Cry1A expression levels in GM maize as the plant reaches maturity would mean that the consequences of exposure will most likely be restricted to the incrop phase of the production cycle. In other cases, the exposure may be restricted to fields where the crop is grown. For example, a transgene introduced to a native plant by outcrossing must be stably introgressed into the genome of the receptor population and persist in order for pollen flow to be a significant route of exposure; if the PF establishes that introgression is not possible, the ERA should focus on the fields where the crop is grown.

\section{Conceptual model}

The conceptual model describes a plausible scenario of how harm may arise from use of the GM crop in a way that allows for a characterization of risk. The purpose of the conceptual model is to readily communicate how the ERA will be conducted. Conceptual models take many forms such as simple statements, an outline of activities, flow charts, or diagrams. Conceptual models describe key relationships between the GM plant release and possible environmental consequences of that release. The conceptual model describes the pathway for analysis by setting the problem in perspective and establishing the proposed relationships between exposure and effect.

Vagueness in development of the conceptual model leads to uncertainty in the application of subsequent risk findings. Vagueness may arise from uncertain regulatory policy, where the protection goal is not clearly articulated. The conceptual model should explicitly recognize the assessment endpoints that have been established, i.e., the entities of value and their measurable attributes. The conceptual model can control both variability and uncertainty in the risk assessment by defining boundaries for the assessment such as the region of use, scale of release, or appropriate comparators.

Diagrammatic conceptual models are particularly powerful as shown in Fig. 2 (Sears et al. 2001). In this example, the various elements contributing to exposure and effect are outlined in a way that 


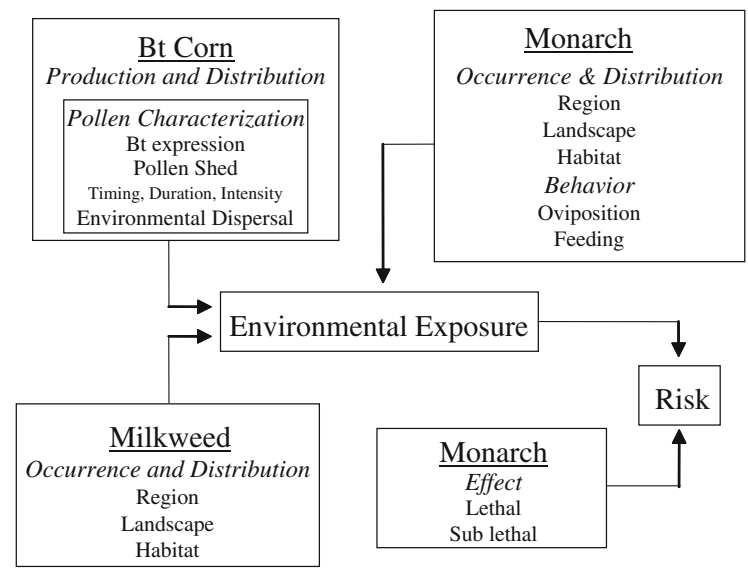

Fig. 2 Example of a conceptual model for ERA (Sears et al. 2001)

describes how data elements and their synthesis are means of evaluating the risks of $\mathrm{Bt}$ maize on monarch butterfly larvae populations.

Analysis plan

Once reasonable scenarios for analysis have been identified and described through conceptual models, they are shaped into an analysis plan. The analysis plan describes the various measures to be used in the assessment, the subsequent characterizations, studies to be conducted, and the appropriate tier for analysis. Importantly, the analysis plan prescribes the manner in which the results should be expressed for risk characterization.

\section{Tiered analysis}

The analytical plan should identify the level of testing and analysis. Well-developed testing schemes for conventional pesticides utilize a tiered approach (Hassan 1998a, b), and this approach should also be considered as a cornerstone for effective GM crop ERA. Tiered testing first addresses broad questions using simple experimental designs with unambiguous outcomes that conservatively cast projections; i.e., appropriately conceptualized early tier assessments should have a low rate of false-negative risk determination but may well have a high rate of false-positives, which will necessitate higher tier assessments.

Problem formulation should seek hypotheses that can be tested in a tiered fashion, because corroboration of hypotheses of no harm when tested with conservative assumptions provides high confidence of low risk. Subsequent tests may refine earlier studies or may progress to studies that are more realistic and complex. These subsequent tests are triggered when the risk assessment requires that more exacting probabilities of exposure and their consequence are needed for decision-making. Since a given tier of testing is only prompted by the risk assessment, the iterative approach effectively focuses consideration to the most relevant concerns and conserves time and resources.

The usefulness of tiered testing and assessment for GM plants is being increasingly recognized (Dutton et al. 2003; Garcia-Alonso et al. 2006; Romeis et al. 2008). Published schemes vary in their specifics such as the number of tiers and the nature of tests, but all recognize the critical nature of tiered approaches to iteratively address risk in a manner consistent with the level of concern and the uncertainty in the assessment.

\section{Measurement endpoints}

The analysis plan must establish a relevant consequence of exposure consistent with the protection goals articulated in the problem context. This represents measurable effects to an environmental entity of value or its surrogate in response to a changed attribute of the GM plant to which the entity is exposed, where the attribute represents the transgenic protein or some other change of importance. The expression of the measurement endpoint must be consistent with the described route, frequency, duration, and intensity of exposure for the attribute relative to the entity of value.

Most assessment endpoints are not measured directly; instead, other characteristics called measurement endpoints are determined (USEPA 1998). For GM plant ERAs, a measurement endpoint is a measurable response to the changed attribute of the plant that is related to the assessment endpoint. For instance, this may be an acute lethal concentration resulting in the death of $50 \%$ of the test organisms $\left(\mathrm{LC}_{50}\right)$, or a chronic No Observable Adverse Effect Level (NOAEL) measured for the entity of value or its surrogate, or altered fecundity in a receptor population.

Often the analysis plan will also be concerned with measures of system or receptor characteristics. These 
are characteristics of the ecosystem that influence the behavior and location of entities of value relative to the distribution of the GM plant. For example, life history characteristics may affect the degree of exposure or response to the GM plant. Therefore, the analysis plan may involve determining the abundance and distribution of the entity of value at a relevant life stage within a landscape or region, as in the assessment of $\mathrm{Bt}$ maize risk to the monarch butterfly population (Sears et al. 2001).

\section{Risk formulation}

The analysis plan will establish the appropriate risk formulation to be considered in the risk characterization. The risk formulation represents the way the exposure measurement is related to the effect measurement. In cases in which the assessment is quantitative, the simplest form of risk formulation may be a ratio or risk quotient (RQ) of exposure to effect measurements, for instance, $R Q=$ Expected Environmental Concentration/LC $\mathrm{L}_{50}$, (Wolt et al. 2003). In some instances, it will be possible to develop a more fully probabilistic formulation of risk (Sears et al. 2001). Not all risk formulations result in quantifiable risk estimates; qualitative descriptors (e.g., negligible, low, high) of risk may be used.

With respect to GM plants, the risk formulation is frequently a qualitative, weight of evidence consideration based on observations relative to a comparator; that is, a determination as to whether the environmental risk associated with the GM plant is no greater than the risk associated with the conventional plant. Quantitative descriptions can be used when assessing risk for specific introduced proteins. For instance, when considering a protein toxin expressed in a GM plant, a threshold for concern arising from a first-tier study would be biologically significant increased mortality for a non-target organism exposed to the purified protein as compared to a no-toxin control. If this was shown for the first-tier study, refinement of effects thresholds or higher tier studies under more realistic exposure conditions (for example, using GM plant material rather than the purified protein) would be necessary in order to adequately address uncertainties regarding the environmental relevance of effects on non-target organisms.

Ideally, an established regulatory threshold for concern (decision criterion) defines both the appropriate risk formulation as well as the result that would trigger regulatory concern. A pro forma decision criterion is seldom found in the case of GM plants. Decision criteria are determinations within the ERA regarding further analysis of the risk hypotheses. The decision criteria will relate to the measurement of the harm (an adverse consequence of exposure or an accepted surrogate measure) and the risk (the manifestation of harm resulting from the exposure that occurs). An example of a decision criterion used in a first-tier, non-target ERA is a meaningful effect to the test organism at $10 \times$ the reasonably anticipated exposure (Rose 2006). In determining a decision criterion, the analytical plan must consider the sufficiency of surrogates as predictors of harmful effects.

\section{Uncertainty}

Uncertainty is inherent to risk analysis and can take multiple forms including incomplete knowledge, variability, or use of language that is vague, ambiguous or under-specified (Morgan and Henrion 1990). Uncertainty that is ignored in the PF stage has the potential to be propagated throughout the risk assessment process. For instance, poorly defined harm or inappropriate assessment endpoints may misdirect research or regulatory effort, and may even lead to the imposition of unnecessary controls to reduce risk. Regardless of its form, uncertainty must be explicitly addressed in the ERA and the PF should describe the approach to dealing with uncertainty. The previously described concept of a tiered system for both testing and assessment is critical for dealing with uncertainties within the ERA in a conservative manner. For instance, a finding of no harm under worse-case assumptions should provide reasonable certainty that there will be low risk to the environmental entity of value under more realistic conditions of exposure.

The PF should prescribe validated test systems as much as possible and utilize conservative dosing strategies consistent with the exposure scenario being evaluated. There are opportunities to address uncertainty through the use of conservatively cast assumptions within each tier of studies and assessment. Also, since GM plants are assessed through a lines-ofevidence approach, it is important that the findings of any one element of the analysis correspond to other 
evidence. The use of comparative assessment is a further aspect of the ERA that acts to reduce uncertainty.

The outcome of any ERA is subject to reinvestigation based on new information arising from many sources. For a particular PF, consideration should be given to the state of uncertainty during development of the analytical plan. For instance, initial testing might identify potential risks that are considered significant but are, in fact, negligible (false positives) or disregard potential risks that are considered negligible but are, in fact, significant (false negatives). Subsequent research may identify risks that are indeed significant and unexpected (surprises). Problem definition attempts to eliminate false negatives and surprises by conservatively assuming in the first instance worst-case exposure scenarios. Consequently, ERAs are likely to include characterization of a number of false-positive risks. The issue relative to false positives is the need for excessive resources to assess negligible risks; this can be addressed through the use of a staged protocol that iterates through tiers. The iterative nature of the ERA allows for the problem to be reformulated for further analysis so as to better address residual uncertainty from earlier stages of analysis.

\section{Summary and recommendations}

Environmental risk assessment is an analytical approach with common elements practiced in various regulatory regimes. Problem formulation defines the goals, objectives, and scope of the ERA in a way that condenses a multitude of concerns within a broadly stated statutory need into a focused problem relevant to the specific GM plant and release being considered. As such, PF is the juncture at which the problem context for risk management is transformed through problem definition into an analytical plan including relevant exposure scenarios and potential consequences (harm) arising from those scenarios. Formal definition of the ERA through a properly executed PF assures the relevance of the risk assessment outcomes for the purpose of decision-making.

In this paper, we have described a framework for $\mathrm{PF}$ and have identified opportunities for strengthening the ERA of GM plants. Adoption of a consistent process for undertaking ERAs, using PF as a focusing step, along with the use of common terminology provides an opportunity to achieve an appropriate degree of uniformity and harmonization in approaches to ERA globally. Critical to the success of ERA is the definition in the PF of clearly articulated pathways for analysis and a distinct process for tiered analysis that matches the level of data generation and synthesis to the nature of concern, degree of uncertainty, and environmental scope of the case being considered. Implicit in this process is reliance on familiarity, experience, and existing knowledge in guiding the extent of analysis required to address questions of risk.

Harmonization of the PF and ERA process will strengthen the ability of ERAs to answer the appropriate questions necessary for risk management decision making and increase the acceptance of the ERA process globally. While adopting a consistent ERA process may not ensure global acceptance of a particular ERA, it will enhance understanding and increase the ease of evaluation of ERAs conducted within different contexts. Adoption of PF is a critical first step for providing transparency in developing and communicating problems that are relevant to analysis with an ERA framework.

Acknowledgments The authors thank the ILSI Research Foundation and CropLife International for their support for this project. David S. Heron, USDA-APHIS, contributed to the expert group deliberation, July 23-24 2007, which led to the formulation of this manuscript. Funding was provided by CropLife International through a grant to the ILSI Research Foundation.

Open Access This article is distributed under the terms of the Creative Commons Attribution Noncommercial License which permits any noncommercial use, distribution, and reproduction in any medium, provided the original author(s) and source are credited.

\section{References}

Australia (2000) Australia Gene Technology Act 2000, http:// www.frli.gov.au/ComLaw/Legislation/ActCompilation1.nsf/ 0/0A2F6253DBF1CBE7CA257313000E8674/\$file/Gene Technology2000_WD02.pdf

Dutton A, Romeis J, Bigler F (2003) Assessing the risks of insect resistant transgenic plants on entomophagous arthropods: Bt-maize expressing Cryl $1 \mathrm{Ab}$ as a case study. BioControl 48:611-636

EFSA (2006) Guidance document of the Scientific Panel on Genetically Modified Organisms for the risk assessment 
of genetically modified plants and derived food and feed. EFSA J 99:1-100

Ellstrand N, Prentice J, Hancock J (1999) Gene flow and introgression from domesticated plants into their wild relatives. Ann Rev Ecol Sys 30:539-563

Garcia-Alonso M, Jacobs E, Raybould A, Nickson TE, Sowig P, Willekens H, Van der KP, Layton R, Amijee F, Fuentes AM, Tencalla F (2006) A tiered system for assessing the risk of genetically modified plants to non-target organisms. Environ Biosafety Res 5(2):57-65

Hassan S (1998a) The initiative of the IOBC/WPRS working group on pesticides and beneficial organism. In: Haskell P, McEwan P (eds) Ecotoxicology: pesticides and beneficial organisms. Kluwer Academic Publishers, Dordrecht, pp 22-27

Hassan S (1998b) Standard laboratory methods to test the sideeffects of pesticides. In: Haskell P, McEwan P (eds) Ecotoxicology: pesticides and beneficial organisms. Kluwer Academic Publishers, Dordrecht, pp 71-79

Hayes KR, Gregg PC, Gupta VV, Jessop R, Lonsdale WM, Sindel B, Stanley J, Williams CK (2004) Identifying hazards in complex ecological systems. Part 3: Hierarchical Holographic Model for herbicide tolerant oilseed rape. Environ Biosafety Res 3(2):109-128

Hayes KR, Kapuscinski A, Danna G, Sifa L. (2007). Overview of the risk assessment framework. In: Kapuscinski A, Hayes KR, Sifa L (eds) Environmental risk assessment of Genetically Modified Organisms, Volume 3: methodologies for transgenic fish. CABI Publishing, Wallingford

Hill RA (2005) Conceptualizing risk assessment methodology for genetically modified organisms. Environ Biosafety Res 4(2):67-70

IPPC (2001) Apr. GMOs, biosafety and invasive species, Third Session of the Interim Commission on Phytosanitary Measures, 02-06 April 2001. Food and Agriculture Organization, Rome, Italy

Johnson KL, Raybould AF, Hudson MD, Poppy GM (2007) How does scientific risk assessment of GM crops fit within the wider risk analysis? Trends Plant Sci 12(1):1-5

Morgan M, Henrion M (1990) Uncertainty: a guide to dealing with uncertainty in quantitative risk and policy analysis. Cambridge University Press, Cambridge

New Zealand (1996) Hazardous substances and new organisms Act 1996, http://www.legislation.govt.nz/act/public/1996/ 0030/latest/DLM381222.html

Nickson TE (2008) Planning environmental risk assessment for genetically modified crops: problem formulation for stress-tolerant crops. Plant Physiol 147(2):494-502
OECD (2003) Descriptions of Selected Key Generic Terms Used in Chemical Hazard/Risk Assessment, OECD Series on Testing and Assessment Number 44, ENV/JM/ MONO(2003)15, Environment Directorate, Joint Meeting of the Chemicals Committee and the Working Party on Chemicals, Pesticides and Biotechnology. Organisation for Economic Co-operation and Development, France

Raybould A (2006) Problem formulation and hypothesis testing for environmental risk assessments of genetically modified crops. Environ Biosafety Res 5(3):119-125

Raybould A (2007) Ecological versus ecotoxicological methods for assessing the environmental risks of transgenic crops. Plant Sci 173:589-602

Raybould A, Cooper I (2005) Tiered tests to assess the environmental risk of fitness changes in hybrids between transgenic crops and wild relatives: the example of virus resistant Brassica napus. Environ Biosafety Res 4(3):127140

Romeis J, Bartsch D, Bigler F, Candolfi MP, Gielkens MM, Hartley SE, Hellmich RL, Huesing JE, Jepson PC, Layton R, Quemada H, Raybould A, Rose RI, Schiemann J, Sears MK, Shelton AM, Sweet J, Vaituzis Z, Wolt JD (2008) Assessment of risk of insect-resistant transgenic crops to nontarget arthropods. Nat Biotechnol 26(2):203-208

Rose RI (2006) Tier-based testing for the effects of proteinaceous insecticidal plant-incorporated protectants on nontarget arthropods in the context of regulatory risk assessments. IOBC WPRS Bull 29(5):143-150

Sears MK, Hellmich RL, Stanley-Horn DE, Oberhauser KS, Pleasants JM, Mattila HR, Siegfried BD, Dively GP (2001) Impact of Bt corn pollen on monarch butterfly populations: a risk assessment. Proc Natl Acad Sci USA 98(21):11937-11942

Standards Australia (2004) Risk management AS/NZS 4360. Sydney and Wellington

Suter GW (2000) Generic assessment endpoints are needed for ecological risk assessment. Risk Anal 20(2):173-178

USEPA (1992) Framework for ecological risk assessment, EPA/630/R-92/001. Risk Assessment Forum; Report nr EPA/630/R-92/001, Washington, DC

USEPA (1998) Guidelines for ecological risk assessment, EPA/630/R-95-002F. Report nr EPA/630/R-95-002F

Wolt JD, Peterson RK, Bystrak P, Meade T (2003) A screening level approach for non-target insect risk assessment: transgenic $\mathrm{Bt}$ corn pollen and the monarch butterfly (Lepidoptera: Danaiidae). Environ Entomol 32:237-246 